\title{
BOUNDS ON INTEGRALS WITH APPLICATIONS TO RELIABILITY PROBLEMS ${ }^{1}$
}

\author{
By Richard E. Barlow \\ University of California, Berkeley
}

1. Summary. Certain statistical problems in life testing and reliability lead to the consideration of minimizing and maximizing certain integrals of the form

$$
\int_{0}^{\infty} \phi(x, \bar{F}(x)) d x, \quad \bar{F}(x)=1-F(x),
$$

under the assumption that the distribution function $F$ has increasing hazard rate and that one or two moments are given. The minimizing (maximizing) distributions for the special cases considered are members of the extremal class considered by A. Marshall and the author [2]. Bounds on the expected values of the minimum and maximum order statistics as well as the operating characteristics of replacement policies based on age are obtained under these assumptions.

2. Statistical motivation. For many statistical problems in life testing and reliability it is natural to assume that the life distribution $F\left(F\left(0^{-}\right)=0\right)$ has increasing hazard rate (is IHR). If $F$ has density $f$, then $F$ is IHR if and only if $f(x) /[1-F(x)]$ is nondecreasing in $x$. In general $F$ is said to be IHR if and only if $\ln [1-F(x)]$ is concave where finite. This property is enjoyed by most of the commonly used life distributions; e.g.,

$$
\text { Weibull: } \begin{aligned}
f(x) & =\lambda \alpha x^{\alpha-1} \exp \left(-\lambda x^{\alpha}\right), & & x \geqq 0, \\
& =0, & & \text { elsewhere, }
\end{aligned}
$$

for $\alpha \geqq 1, \lambda>0$, and

$$
\text { Gamma: } \begin{aligned}
f(x) & =\left[\left(\lambda^{\alpha} x^{\alpha-1}\right) /(\Gamma(\alpha))\right] \exp (-\lambda x), & & x \geqq 0, \\
& =0, & & \text { elsewhere, }
\end{aligned}
$$

for $\alpha \geqq 1, \lambda>0$. Distributions $F$ for which $\ln [1-F(x)]$ is convex on $[0, \infty)$ are called DHR for decreasing hazard rate. Properties of distributions with monotone hazard rate are discussed in [3].

In addition to this assumption, some prior information in the form of the mean $\mu_{1}$, and the variance $\sigma^{2}=\mu_{2}-\mu_{1}^{2}$ of the distribution may be known. Since in the IHR case, $\sigma / \mu_{1} \leqq 1$ with equality only for exponential distribution, an estimate of the coefficient of variation $\sigma / \mu_{1}$ may represent the statistician's belief in the amount of departure of the failure distribution from exponentiality.

With the mean and variance assumed known we consider the problem of

Received 4 April 1964.

1 This research has been partially supported by the Office of Naval Research under Contract nonr-222(83) with the University of California. Reproduction in whole or in part is permitted for any purpose of the United States Government. 
minimizing and maximizing certain integrals of the form

$$
\int_{0}^{\infty} \phi(x, \bar{F}(x)) d x
$$

where $F$ is IHR. Integrals of such functions have been considered by Karlin [7], Rustagi [11] and others for general distributions. (See [11] for additional references.) A related but more specialized problem is that of minimizing (maximizing) integrals of the form $\int_{0}^{\infty} \rho(x) d F(x)$ assuming that certain moments of $F$ are specified, (cf. Theorem 4.1). Assuming no conditions other than the moment conditions, this problem has been solved by H. P. Mulholland and C. A. Rogers [10]. Their methods as well as those of many others (e.g. Karlin and Shapley [9]) use the fact that the class of all distributions subject to specified moment conditions are closed under convex combinations. However, the class of IHR distributions is not closed under convex combinations ([3], p. 381) and, therefore, we cannot use the classical methods. Instead we consider very special cases using the methods of [2]. However, we do obtain fairly explicit bounds which can be easily machine computed. The resul ts of Section 3 follow from a theorem of Ky Fan and G. G. Lorentz. The main results of the paper are in Section 4.

Integrals such as (2.1) often arise in statistical applications. For example, let $U_{1} \leqq U_{2} \leqq \cdots \leqq U_{n}$ denote $n$ ordered observations from a population with IHR cdf $F$. Let $W_{n}=U_{n}-U_{1}$ denote the sample range. Then we may seek to minimize (maximize)

$$
E\left(W_{n}\right)=\int_{0}^{\infty}\left[1-F(x)^{n}-(\bar{F}(x))^{n}\right] d x
$$

where $\bar{F}(x)=1-F(x)$. The integrand is of the form $\phi(x, y)$ where

$$
\phi(x, y)=1-(1-y)^{n}-y^{n}
$$

is concave in $y$.

For another example, consider a replacement policy of the form: Replace at time $t$ or at failure, whichever occurs first. We may then seek to minimize (maximize) the following integrals:

$$
\int_{0}^{t} \bar{F}(x) d x, \quad \bar{F}(x)=1-F(x),
$$

i.e., the expected time between removals for either a failed or nonfailed item.

$$
\int_{0}^{t} \vec{F}(x) d x / F(t)
$$

i.e., the expected time between failure removals using a replacement policy based on $t$. The reciprocal of this quantity occurs as an upper bound on the renewal function divided by $t,[5]$.

$$
\int_{0}^{t} \bar{F}(x) d x / \bar{F}(t)
$$

i.e., the expected time between planned replacements using a replacement policy based on $t$. A related expression is:

$$
\int_{t}^{\infty} \bar{F}(x) d x / \bar{F}(t)
$$


i.e., the mean residual life of an item aged $t$. Bounds on densities and failure rate functions will be discussed in a future joint paper with A. Marshall.

3. Bounds on integrals when $F$ is IHR (DHR) with specified mean. If $\phi(x, y)$ is sufficiently smooth the problem of minimizing (maximizing) $\int_{0}^{\infty} \phi(x, \bar{F}(x)) d x$ is comparatively simple. To do this we first state a more general result due to Ky Fan and G. G. Lorentz ([6], p. 630). This reference was pointed out by A. W. Marshall. We consider a system of decreasing bounded functions $f_{i}, g_{i}$ where $f_{i} \prec g_{i}, i=1,2, \cdots, n$. The relation $f \prec g$ means that

and

$$
\int_{0}^{x} f(t) d t \leqq \int_{0}^{x} g(t) d t \quad \text { for all } x \geqq 0
$$

$$
\int_{0}^{\infty} f(t) d t=\int_{0}^{\infty} g(t) d t .
$$

Theorem 3.1 (Ky Fan and G. G. Lorentz). If

(i) $f_{i}, g_{i}$ are bounded and decreasing $i=1,2, \cdots, n$;

(ii) $f_{i} \prec g_{i}, i=1,2, \cdots, n$;

(iii) $\phi\left(x, y_{1}, y_{2}, \cdots, y_{n}\right)$ has continuous second derivatives;

(iv) $\partial^{2} \phi / \partial y_{i} \partial y_{j} \geqq 0, \partial^{2} \phi / \partial x \partial y_{i} \leqq 0, i, j=1,2, \cdots, n$; then

$$
\int_{0}^{\infty} \phi\left(x, f_{1}, f_{2}, \cdots, f_{n}\right) d x \leqq \int_{0}^{\infty} \phi\left(x, g_{1}, \cdots, g_{n}\right) d x
$$

when the integrals exist.

Fan and Lorentz actually state this as a corollary and for the interval $[0,1]$. The theorem can be extended to $[0, \infty)$ by limiting arguments assuming that the integrals exist.

Note that if $F_{i}$ is IHR with mean $\mu_{i}$ and $\bar{G}_{i}(x)=e^{-x / \mu_{i}}$ then $\bar{G}_{i} \prec \bar{F}_{i}$ (see [8]). The relation is reversed if $F_{i}$ is DHR with mean $\mu_{i}$. Let

$$
\begin{array}{rlrl}
\bar{G}_{\mu_{i}}(x) & =1, & & 0<x<\mu_{i}, \\
& =0, & x \geqq \mu_{i} .
\end{array}
$$

Then we always have $\bar{F}_{i} \prec \bar{G}_{\mu_{i}}$. Hence assuming $F_{i}$ is IHR and Conditions (iii), (iv) of Theorem 3.1 hold we have

$$
\int_{0}^{\infty} \phi\left(x, \bar{G}_{\mu_{i}}(x)\right) d x \geqq \int_{0}^{\infty} \phi\left(x, \bar{F}_{i}(x)\right) d x \geqq \int_{0}^{\infty} \phi\left(x, e^{-x / \mu_{i}}\right) d x .
$$

Let $X_{1}, X_{2}, \cdots, X_{n}$ denote $n$ independent observations on a random variable $X$ with IHR distribution $F$. Let $U_{1} \leqq U_{2} \leqq \cdots \leqq U_{n}$ denote the associated order statistics. Then

$$
\begin{aligned}
E\left[U_{n}-U_{1}\right] & =\int_{0}^{\infty}\left\{1-[\bar{F}(x)]^{n}-[1-\bar{F}(x)]^{n}\right\} d x \\
& =\int_{0}^{\infty} \phi(x, \bar{F}(x)) d x
\end{aligned}
$$

where $\phi(x, y)=1-y^{n}-(1-y)^{n}$. Theorem 3.1 applies and we have

$$
0 \leqq E\left[U_{n}-U_{1}\right] \leqq \mu_{1} \sum_{k=1}^{n-1} 1 / k
$$

for sharp upper and lower bounds on the expected range. Intuitively, this in- 
equality tells us that observations on IHR random variables tend to cluster together more closely than observations from an exponentially distributed random variable with the same mean. In a similar manner we obtain sharp upper and lower bounds on the expected values of the minimum and the maximumobservations.

$$
\begin{aligned}
\mu_{1} / n \leqq E\left[U_{1}\right] & =\int_{0}^{\infty}[\bar{F}(x)]^{n} d x \leqq \mu_{1} \\
\mu_{1} \leqq E\left[U_{n}\right] & =\int_{0}^{\infty}\left[1-F^{n}(x)\right] d x \leqq \mu_{1} \sum_{1}^{n} 1 / k .
\end{aligned}
$$

Inequalities (3.1) and (3.2) also follow from Corollary 3.1.

Corollary 3.1. If $F_{i}(i=1,2, \cdots, n)$ is IHR (DHR) with means $\mu_{i}, i=$ $1,2, \cdots, n$, then

$$
\int_{0}^{\infty} \alpha x^{\alpha-1} \prod_{i=1}^{n} \bar{F}_{i}(x) d x \geqq(\leqq) \int_{0}^{\infty} \alpha x^{\alpha-1} \prod_{i=1}^{n} \bar{G}_{i}(x) d x
$$

for $0 \leqq \alpha \leqq 1$ and

$$
\int_{0}^{\infty} \alpha x^{\alpha-1}\left[1-\prod_{i=1}^{n} F_{i}(x)\right] d x \leqq(\geqq) \int_{0}^{\infty} \alpha x^{\alpha-1}\left[1-\prod_{i=1}^{n} G_{i}(x)\right] d x
$$

for $\alpha \geqq 1$.

This is a straightforward specialization of Theorem 3.1. In a reliability context, Part (i) says (for $\alpha=1$ ) that the mean life of a series system with IHR components whose means are $\mu_{i}(i=1,2, \cdots, n)$ exceeds the mean life of a series system with exponentially distributed components and means $\mu_{i}(i=1,2, \cdots, n)$. However, just the reverse is true for parallel systems.

Additional properties of order statistics from IHR (DHR) distributions together with applications to life testing will appear in a future paper.

4. Bounds on integrals when $F$ is IHR with specified mean and variance. Assume now that $F$ is IHR with mean $\mu_{1}$ and $\mu_{2}$ specified. In [2] the class of extremal distributions for bounding $F$ were determined. Two rather special members of this class are

$$
\begin{aligned}
1-G_{T_{1}}(x) & =\exp \left(-a_{0} x\right), & & x<T_{1}, \\
& =0, & & x \geqq T_{1},
\end{aligned}
$$

and

$$
\begin{aligned}
1-G_{T_{0}}(x) & =1, & & x \leqq 1- \\
& =\exp \left[-\left(x-T_{0}\right)\left(\mu_{2}-1\right)^{-\frac{1}{3}}\right], & & x \geqq T_{0},
\end{aligned}
$$

where $a_{0}$ and $T_{1}$ are chosen to satisfy the moment conditions.

The method we now use has been employed by Karlin and Novikoff [8] among others.

THEOREM 4.1. If

(i) $F$ is IHR, $F(0)=0$,

(ii) $\mu_{1}=1$ and $\mu_{2}$ are specified,

(iii) $\rho(x)$ is convex, 
then

$$
\int_{0}^{\infty} \rho(x) \bar{G}_{T_{1}}(x) d x \leqq \int_{0}^{\infty} \rho(x) \bar{F}(x) d x \leqq \int_{0}^{\infty} \rho(x) \bar{G}_{T_{0}}(x) d x .
$$

Proof. We know [2] that $\bar{F}(x)-\bar{G}_{T_{0}}(x)$ has exactly two sign changes, say at $x_{1}$ and $x_{2}$. Furthermore, the order of the sign changes is -+- . Define a line $\ell(x)$ so that

$$
\rho\left(x_{1}\right)=\ell\left(x_{1}\right) \text { and } \rho\left(x_{2}\right)=\ell\left(x_{2}\right) .
$$

Since $\rho$ is convex, $\rho(x)-\ell(x)$ changes sign at $x_{1}$ and $x_{2}$ in the order +-+ . Therefore, $[\rho(x)-\ell(x)]\left[\bar{F}(x)-\bar{G}_{T_{0}}(x)\right] \leqq 0$ for all $x$. Integrating on $x$ we obtain the upper bound on $\int_{0}^{\infty} \rho(x) \vec{F}(x) d x$. The lower bound follows in a similar way using $\bar{G}_{T_{1}}(x)$ instead of $\bar{G}_{T_{0}}(x)$.\|

The ordering of sign changes for $\rho(x)-\ell(x)$ is of course reversed when $\rho$ is concave. Using Theorem 4.1 and letting $\rho(x)=x^{r}$ for $r>0$, one can obtain improved bounds on $\mu_{r}(r>2)$ in terms of $\mu_{1}$ and $\mu_{2}$ (cf. Theorem 4.4 of [3]).

The general problem of bounding integrals of the form

$$
\int_{0}^{\infty} \phi(x, \bar{F}(x)) d x
$$

when $F$ is IHR with $\mu_{1}$ and $\mu_{2}$ specified seems quite difficult. However, it is possible to treat many special cases using the properties of the extremal family considered in [2]. In order to do this we repeat some of the notation in [2]. We can assume without loss of generality that $\mu_{1}=1$. It follows that $\mu_{2}$ satisfies $1 \leqq \mu_{2} \leqq 2$. Let

$$
T_{0}=1-\left(\mu_{2}-1\right)^{\frac{1}{2}} \text { and } T_{1}=-a_{0}^{-1} \log \left(1-a_{0}\right),
$$

where $a_{0}$ in $[0,1]$ and $T_{1}$ simultaneously satisfy the following equations:

$$
\int_{0}^{T_{1}} e^{-a_{0} x} d x=\mu_{1}=1, \quad \int_{0}^{T_{1}} x e^{-a_{0} x} d x=\frac{1}{2} \mu_{2} .
$$

In [2] it is shown that solutions exist. Let

$$
\Theta_{3}=\left\{G_{T}: T \geqq T_{1}\right\}
$$

where

$$
\begin{aligned}
1-G_{T}(x) & =1, & & x<\Delta, \\
& =e^{-a(x-\Delta)}, & & \Delta \leqq x \leqq T, T \geqq T_{1}, \\
& =0, & & x \geqq T,
\end{aligned}
$$

and $a, \Delta\left(0 \leqq \Delta \leqq T_{0}\right)$ are chosen to satisfy the moment conditions; i.e.,

$$
\int_{0}^{\infty}\left[1-G_{T}(x)\right] d x=\mu_{1}=1, \quad \int_{0}^{\infty} x\left[1-G_{T}(x)\right] d x=\frac{1}{2} \mu_{2} .
$$

Let

$$
\mathcal{G}_{4}=\left\{G_{T}: T_{0} \leqq T \leqq T_{1}\right\}
$$


where

$$
\begin{aligned}
1-G_{T}(x) & =\exp \left(-a_{1} x\right), & & x \leqq T, \\
& =\exp \left(-a_{1} T-a_{2}(x-T), \quad\right. & x \geqq T, &
\end{aligned}
$$

and $a_{1} \leqq a_{2}$ are chosen to satisfy the moment conditions as before. It is shown in [2] that for $t \geqq 0$

$$
\inf \left[1-G_{T}(t)\right] \leqq 1-F(t) \leqq \sup \left[1-G_{T}(t)\right]
$$

where the extremums are taken over $\mathcal{S}_{3} \cup \mathcal{S}_{4}$. These bounds have been tabulated for selected values of $\mu_{2}\left(1 \leqq \mu_{2} \leqq 2\right)$, [1].

4.1 Expected time between removals. If $X$ has distribution $F$, then $E[\min (X, t)]$ $=\int_{0}^{t} \bar{F}(x) d x$. For an age replacement policy this denotes the expected time between removals using a policy determined by $t$.

Theorem 4.2. If $F$ is IHR, $F(0)=0$ and $\mu_{1}=1$, $\mu_{2}$ are specified, then

$$
\begin{aligned}
& \int_{0}^{t} \bar{F}(x) d x \geqq \inf _{G_{T} \varepsilon \mathcal{G}_{3}} \int_{0}^{t} \bar{G}_{T}(x) d x, \\
& \text { (ii) } \int_{0}^{t} \bar{F}(x) d x \leqq t \text {, } \\
& t \leqq T_{0}=1-\left(\mu_{2}-1\right)^{\frac{1}{2}}, \\
& \leqq \sup _{G_{T} \varepsilon \mathcal{G}_{4}} \int_{0}^{t} \bar{G}_{T}(x) d x, \quad T_{0} \leqq t \leqq T_{1}, \\
& \leqq \mu_{1}-1, \quad t \geqq T_{1} \text {. }
\end{aligned}
$$

All inequalities are sharp.

Proof. Let $F \mathcal{F} \mathcal{F}-\left(\mathcal{G}_{3} \cup \mathcal{G}_{4}\right)$ where $\mathcal{F}$ is the family of all IHR distributions with prescribed moments $\mu_{1}=1$ and $\mu_{2}$. Let $T>T_{1}$ and $s(T)$ be the crossing in $(\Delta, T)$ from above of $1-G_{T}$ by $1-F$. We use crucially the fact that $s(T)$ is continuous in $T$ (see [2]).

CASE 1. $t \leqq s\left(T_{1}\right)$. From the definition of $G_{T_{1}}$ we can see that $\bar{F}(x) \geqq \bar{G}_{T_{1}}(x)$ for $0 \leqq x \leqq s\left(T_{1}\right)$ and hence $\int_{0}^{t} \bar{F}(x) d x \geqq \int_{0}^{t} \bar{G}_{T_{1}}(x) d x$.

CASE $2 . t \geqq s(\infty) . \bar{F}(x)$ crosses $\bar{G}_{T_{0}}(x)$ from above at $s(\infty)$ and $\bar{F}(x) \leqq$ $\bar{G}_{T_{0}}(x)$ for $x>s(\infty)$. Hence $\int_{t}^{\infty} \bar{F}(x) d x \leqq \int_{t}^{\infty} \bar{G}_{T_{0}}(x) d x$ implies $\int_{0}^{t} \bar{F}(x) d x \geqq$ $\int_{0}^{t} \bar{G}_{T_{0}}(x) d x$.

CASE 3. $s\left(T_{1}\right)<t<s(\infty)$. Since $s(T)$ is continuous in $T$, choose $T \geqq T_{1}$ and $\Delta\left(0 \leqq \Delta \leqq T_{0}\right)$ such that $s(T)=t$. In this circumstance $G_{T} \varepsilon G_{3}$. See Fig.

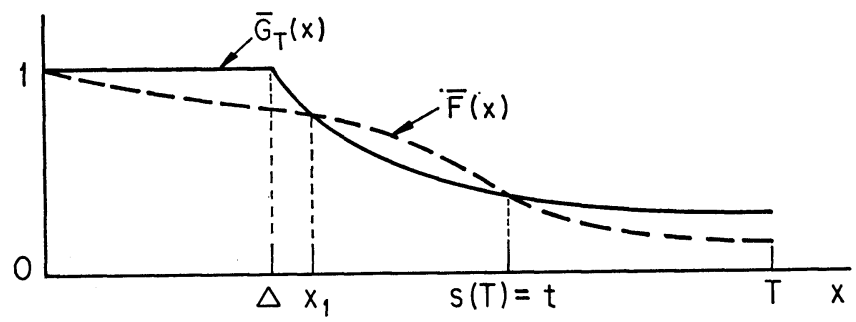

FIG. 4.1 
4.1. Let

$$
\begin{aligned}
\phi_{t}(x) & =1, & & x \leqq t, \\
& =0, & & x>t,
\end{aligned}
$$

and define a line $\ell(x)$ such that $\ell\left(x_{1}\right)=1$ and $\ell(T)=0$. Since clearly

$$
\left[\phi_{t}(x)-\ell(x)\right]\left[\bar{F}(x)-\bar{G}_{T}(x)\right] \geqq 0
$$

for all $x$,

$\int_{0}^{\infty}\left[\phi_{t}(x)-\ell(x)\right]\left[\bar{F}(x)-\bar{G}_{T}(x)\right] d x=\int_{0}^{t} \bar{F}(x) d x-\int_{0}^{t} \bar{G}_{T}(x) d x \geqq 0$.

This proves (i).

To prove (ii) we first note that $\bar{F}(x) \leqq \bar{G}_{T_{0}}(x)=1$ for $0 \leqq x \leqq T_{0}$ and hence $\int_{0}^{t} \bar{F}(x) d x \leqq t$ for $t \leqq T_{0}$, is sharp. Also, $\bar{F}(x) \geqq \bar{G}_{T_{1}}(x)$, for $x \geqq T_{1}$, implies $\int_{0}^{t} \bar{F}(x) d x \leqq \int_{0}^{t} \bar{G}_{T_{1}}(x) d x=\mu_{1}=1, t \geqq T_{1}$ is sharp. To complete the proof we need the function $u(T)$ defined for $T_{0} \leqq T \leqq T_{1}$. Let $u(T)$ be the crossing in $[T, \infty]$ from below of $\bar{G}_{T}$ by $\bar{F} ; u(T)$ always exists (see [2]). We use crucially the fact that $u(T)$ varies continuously from $u\left(T_{0}\right)>T_{0}$ to $u\left(T_{1}\right)=T_{1}$.

CASE 1a. $t \leqq u\left(T_{0}\right)$. Clearly $\bar{F}(x) \leqq \bar{G}_{T_{0}}(x)$ for $x \leqq u\left(T_{0}\right)$ implies

$$
\int_{0}^{t} \bar{F}(x) d x \leqq \int_{0}^{t} \bar{G}_{T_{0}}(x) d x .
$$

CASE 2a. $T_{0} \leqq u\left(T_{0}\right)<t<u\left(T_{1}\right)=T_{1}$. By continuity of $u(T)$ we can choose $T$ such that $u(T)=t$. In this circumstance $G_{T} \varepsilon G_{4}$. See Fig. 4.2. If $\bar{F}(x) \leqq \bar{G}_{T}(x)$ for $x \leqq t$, the proof is obvious. Hence suppose $\bar{F}(x)$ crosses $\bar{G}_{T}(x)$ from above at $x_{1}$. If $x_{2}=\infty$, the proof is also obvious. Hence suppose $x_{2}<\infty$. Let

$$
\begin{aligned}
\phi_{t}(x)=1, & x \leqq t, \\
& =0, \quad x>t,
\end{aligned}
$$

and construct a line $\ell(x)$ so that $\ell\left(x_{1}\right)=1$ and $\ell\left(x_{2}\right)=0$. Since

$$
\left[\phi_{t}(x)-\ell(x)\right]\left[\bar{F}(x)-\bar{G}_{T}(x)\right] \leqq 0
$$

for all $x$ we have

$$
\int_{0}^{\infty}\left[\phi_{t}(x)-\ell(x)\right]\left[\bar{F}(x)-\bar{G}_{T}(x)\right] d x=\int_{0}^{t} \bar{F}(x) d x-\int_{0}^{t} \bar{G}_{T}(x) d x \leqq 0 . \|
$$

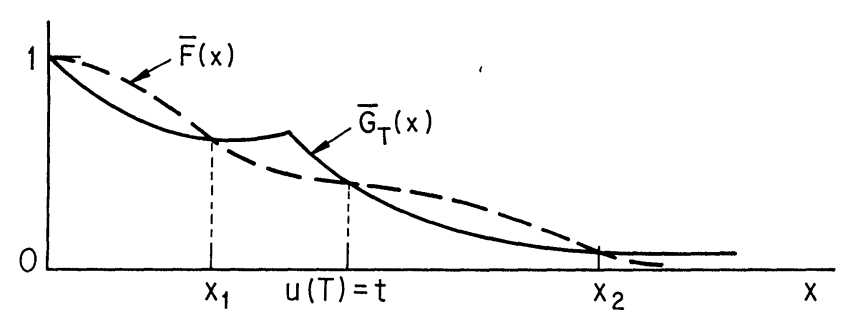

FIg. 4.2 
Although the above bounds must be computed numerically, they can be easily programmed because of the simple form of the extremal distributions.

If $\mu_{1}$ is the only moment specified ( $F$ IHR), then

$$
\begin{aligned}
\int_{0}^{t} \bar{F}(x) d x & \geqq \mu_{1}\left[1-e^{-t / \mu_{1}}\right], \\
\int_{0}^{t} \bar{F}(x) d x & \leqq t, \quad t<\mu_{1}, \\
& \leqq \mu_{1}, \quad t \geqq \mu_{1} .
\end{aligned}
$$

All inequalities are sharp. The lower bound follows from the fact that $\bar{F}(x)$ crosses $e^{-x / \mu_{1}}$ at most once and from above. The inequality is clear for small $t$. If it were ever violated, then $a$ fortiori it would also be violated for $t=+\infty$ which is impossible.

4.2 Additional bounds. We now present additional bounds on quantities related to age replacement problems. The mean residual life of an item aged $t$ is

$$
\int_{t}^{\infty} \bar{F}(x) d x / \bar{F}(t)
$$

If $F$ is IHR, this ratio is necessarily nonincreasing in $t$.

THEOREM 4.3. If $F$ is IHR, $F(0)=0$ and $\mu_{1}=1, \mu_{2}$ are specified then

$$
\text { (i) } \begin{aligned}
\int_{t}^{\infty} \bar{F}(x) d x / \bar{F}(t) & \geqq \mu_{1}-t, & & t \leqq T_{0}, \\
& \geqq \inf _{G_{T} \varepsilon G_{4}}\left[\int_{t}^{\infty} \bar{G}_{T}(x) d x / \bar{G}_{T}(t)\right], & & T_{0} \leqq t \leqq T_{1}, \\
& \geqq 0, & & t>T_{1}, \\
\text { (ii) } \quad \int_{t}^{\infty} \bar{F}(x) d x / \bar{F}(t) & \leqq \mu_{1}=1, & & t=0, \\
& \leqq \sup _{G_{T} \varepsilon \xi_{3}}\left[\int_{t}^{\infty} \bar{G}_{T}(x) d x / \bar{G}_{T}(t)\right], & & 0<t<\infty, \\
& \leqq\left(\mu_{2}-1\right)^{\frac{3}{2}}, & & t=+\infty .
\end{aligned}
$$

All inequalities are sharp.

The proof follows by an examination of cases and application of the methods of Theorem 4.2 .

It is easy to show that if only $\mu_{1}$ is specified ( $F$ IHR), then the following inequalities are sharp.

$$
\begin{aligned}
\int_{t}^{\infty} \bar{F}(x) d x / \bar{F}(t) & \geqq \mu_{1}-t, & & t \leqq \mu_{1}, \\
& \geqq 0, & & t>\mu_{1}, \\
\int_{t}^{\infty} \bar{F}(x) d x / \bar{F}(t) & \leqq \mu_{1} . & &
\end{aligned}
$$

Expected time between failure removals. If an item is replaced either at age $t$ or at failure, whichever occurs first, then the expected time between failure removals will be

$$
\int_{0}^{t} \bar{F}(x) d x / F(t) .
$$

This ratio is nonincreasing in $t$ if $F$ is IHR. This ratio also occurs in a bound on the renewal function. Suppose $F$ is IHR and $N(t)$ is the number of renewals in $[0, t]$ for an associated renewal process. Then it can be shown [5] that

$$
E[N(t)] \leqq t F(t) / \int_{0}^{t} \bar{F}(x) d x \leqq t / \mu_{1} .
$$


It is therefore of some interest to obtain bounds on this ratio.

THEOREM 4.4. If $F$ is IHR, $F(0)=0$ and $\mu_{1}=1, \mu_{2}$ are specified, then

$$
\begin{array}{rlrl}
\int_{0}^{t} \bar{F}(x) d x / F(t) & \geqq \inf _{G_{T \varepsilon} \Theta_{3}}\left[\int_{0}^{t} \bar{G}_{T}(x) d x / G_{T}(t)\right], & & t \leqq \mu_{1}=1, \\
& \geqq \inf _{G_{T \varepsilon} \varepsilon \cup \Theta_{4}}\left[\int_{0}^{t} \bar{G}_{T}(x) d x / G_{T}(t)\right], & & \mu_{1} \leqq t<T_{1}, \\
& \geqq \mu_{1}, & t \geqq T_{1}, \\
\int_{0}^{t} \bar{F}(x) d x / F(t) & \leqq \infty, & t \leqq T_{0}=1-\left(\mu_{2}-1\right)^{\frac{1}{2}} \\
& \leqq \sup _{G_{T \varepsilon} \oint_{4}}\left[\int_{0}^{t} \bar{G}_{T}(x) d x / G_{T}(t)\right], & & T_{0} \leqq t \leqq T_{1} \\
& \leqq \sup _{G_{T \varepsilon} \oint_{3}}\left[\mu_{1} / G_{T}(t)\right], & & t>T_{1} .
\end{array}
$$

(ii) $\int_{0}^{t} \bar{F}(x) d x / F(t) \leqq \infty$,

All inequalities are sharp.

We omit the proof.

The asymptotic expected cost per unit time under an age replacement policy where an item is replaced either at failure or at time $t$ whichever occurs first is [cf. [7] p. 68]

$$
C(t)=\left[\left(c_{1}-c_{2}\right) F(t)+c_{2}\right] / \int_{0}^{t} \bar{F}(x) d x, \quad\left(c_{1} \geqq c_{2}\right) .
$$

The methods of Theorem 4.4 will also provide sharp bounds on this function.

If only $\mu_{1}$ is specified, ( $F$ IHR), then the following inequalities are sharp:

$$
\begin{aligned}
\int_{0}^{t} \bar{F}(x) d x / F(t) & \geqq \mu_{1}, & & \text { for all } t, \\
\int_{0}^{t} \bar{F}(x) d x / F(t) & \leqq \infty, & & t \leqq \mu_{1}, \\
& \leqq \mu_{1} /\left(1-e^{-w t}\right), & & t>\mu_{1},
\end{aligned}
$$

where $w$ depends on $t$ and is determined by $\int_{0}^{t} e^{-w x} d x=\mu_{1}=1$. The first inequality is attained by the exponential. The second inequality is attained by the degenerate and exponentials truncated on the right.

Expected time between planned replacements. If an item is replaced either at time $t$ or at failure, whichever occurs first, then the expected time between planned replacements will be

$$
\int_{0}^{t} \bar{F}(x) d x / \bar{F}(t) .
$$

This ratio is always nondecreasing in $t$.

Theorem 4.5. If $F$ is IHR, $F(0)=0$ and $\mu_{1}=1, \mu_{2}$ are specified, then

$$
\begin{aligned}
& \int_{0}^{t} \bar{F}(x) d x / \bar{F}(t) \geqq t, \quad 0 \leqq t \leqq T_{0}, \\
& \geqq \inf _{\mathcal{G}_{3} \cap_{\mathcal{S}_{4}}}\left[\int_{0}^{t} \bar{G}_{T}(x) d x / \bar{G}_{T}(t)\right], \quad T_{0} \leqq t \leqq T_{1}, \\
& \geqq \inf _{\mathcal{G}_{3}}\left[\int_{0}^{t} \bar{G}_{T}(x) d x / \bar{G}_{T}(t)\right], \quad t>T_{1}, \\
& \int_{0}^{t} \bar{F}(x) d x / \bar{F}(t) \leqq \sup _{S_{3}}\left[\int_{0}^{t} \bar{G}_{T}(x) d x / \bar{G}_{T}(t)\right], \quad 0 \leqq t \leqq T_{0}, \\
& \leqq \sup _{\oint_{3} \cap \varrho_{4}}\left[\int_{0}^{t} \bar{G}_{T}(x) d x / \bar{G}_{T}(t)\right], \quad T_{0}<t \leqq T_{1}, \\
& \leqq \infty, \quad t>T_{1} \text {. }
\end{aligned}
$$


All inequalities are sharp.

We omit details of the proof.

If the only moment specified is the mean $\mu_{1}(F$ IHR), then the following inequalities are sharp.

$$
\begin{aligned}
\int_{0}^{t} \bar{F}(x) d x / \bar{F}(t) & \geqq t, & & t \leqq \mu_{1}, \\
& \geqq \inf _{w}\left[\int_{0}^{t} \bar{G}_{w}(x) d x / \bar{G}_{w}(t)\right], & & t>\mu_{1}, \\
\int_{0}^{t} \bar{F}(x) d x / \bar{F}(t) & \leqq \sup _{0 \leqq \Delta \leqq \mu_{1}}\left[\int_{0}^{t} \bar{G}_{\Delta}(x) d x / \bar{G}_{\Delta}(t)\right], & & t<\mu_{1}, \\
& \leqq+\infty, & & t \geqq \mu_{1},
\end{aligned}
$$

where

$$
\begin{aligned}
\bar{G}_{w}(x) & =e^{-w x}, & & x<T, \\
& =0, & & x \geqq T,
\end{aligned}
$$

$w$ and $T$ are chosen to satisfy $\int_{0}^{T} e^{-w x} d x=\mu_{1}$ and

$$
\begin{aligned}
\bar{G}_{\Delta}(x) & =1, & 0 \leqq x \leqq \Delta, \\
& =\exp \left[-(x-\Delta) /\left(\mu_{1}-\Delta\right)\right], & x \geqq \Delta,
\end{aligned}
$$

$\left(0 \leqq \Delta \leqq \mu_{1}\right)$. The proof is similar to previous proofs and uses the methods of [2], pp. 1258-1261.

Acknowledgment. This paper represents an extension of a previous paper [2] with Albert Marshall to whom I would like to acknowledge a great indebtedness.

\section{REFERENCES}

[1] Barlow, R. E. and Marshall, A. W. (1963). Tables of bounds for distributions with monotone hazard rate. Boeing Scientific Research Laboratories Document DI-82-0249.

[2] Barlow, R. E. and Marshall, A. W. (1964). Bounds for distributions with monotone hazard rate, II. Ann. Math. Statist. 35 1258-1274.

[3] Barlow, R. E., Marshall, A. W., and Proschan, F. (1963). Properties of distributions with monotone hazard rate. Ann. Math. Statist. 34 375-389.

[4] Barlow, R. E. and Proschan, F. (1962). Planned replacement. Studies in Applied Probability and Management Science. Stanford Univ. Press. 63-87.

[5] Barlow, R. E. and Proschan, F. (1964). Comparison of replacement policies and renewal theory implications. Ann. Math. Statist. 35 577-589.

[6] Fan, K. and Lonentz, G. G. (1954). An integral inequality. Amer. Math. Monthly $61626-631$.

[7] Karlin, S. (1959). Mathematical Methods and Theory in Games, Programming, and Economics, 2. Addison-Wesley, Reading. 210-214.

[8] Karlin, S. and Novikoff, A. (1963). Generalized convex inequalities. Pacific J. Math. 13 1251-1279.

[9] Karlin, S. and Shapley, L. S. (1953). Geometry of moment spaces. Memoir No. 12 of the Amer. Math. Soc.

[10] Mulholland, H. P. and Rogers, C. A. (1958). Representation theorems for distribution functions. Proc. London Math. Soc. 8 No. 30.

[11] Rustagi, J. S. (1957). On minimizing and maximizing a certain integral with statistical applications. Ann. Math. Statist. 28 309-328. 